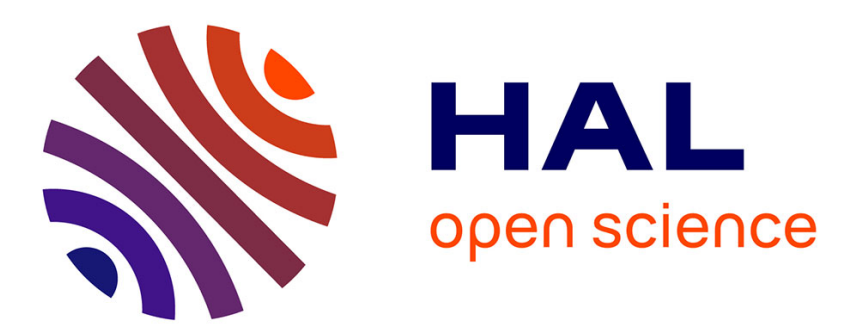

\title{
Control of the light-response in supramolecular metallopolymeric gels by tuning the coordination metal
}

Etienne Borré, Jean-François Stumbé, Stéphane Bellemin-Laponnaz, Matteo

Mauro

\section{- To cite this version:}

Etienne Borré, Jean-François Stumbé, Stéphane Bellemin-Laponnaz, Matteo Mauro. Control of the light-response in supramolecular metallopolymeric gels by tuning the coordination metal. Chemical Communications, 2017, 53 (59), pp.8344-8347. 10.1039/C7CC03516K . hal-03551437

\author{
HAL Id: hal-03551437 \\ https://hal.science/hal-03551437
}

Submitted on 1 Feb 2022

HAL is a multi-disciplinary open access archive for the deposit and dissemination of scientific research documents, whether they are published or not. The documents may come from teaching and research institutions in France or abroad, or from public or private research centers.
L'archive ouverte pluridisciplinaire HAL, est destinée au dépôt et à la diffusion de documents scientifiques de niveau recherche, publiés ou non, émanant des établissements d'enseignement et de recherche français ou étrangers, des laboratoires publics ou privés. 


\section{Control of the photoswitching properties in supramolecular metallopolymeric gels by tuning the coordination metal}

Received 00th January 20xx, Accepted 00th January 20xx

DOI: $10.1039 / x 0 \times x 00000 x$

\author{
Etienne Borré, ${ }^{\mathrm{a}, \mathrm{b}}$ Jean-François Stumbé, ${ }^{\mathrm{c}}$ Stéphane Bellemin-Laponnaz ${ }^{*}, \mathrm{~b}, \mathrm{~d}$ and Matteo Mauro ${ }^{*}, \mathrm{a}, \mathrm{d}$
}

Two novel supramolecular metallo-heteropolymers bearing a photo-isomerizable telechelic bis-terpyridine ligand and either $\mathrm{Fe}$ (II) or $\mathrm{Co}$ (II) coordination metals were synthesized. Both polymers induced gelation of organic solvents at concentration as low as $\mathbf{0 . 1 2}$ wt.\% yielding thixotropic gels. Judicious choice of electronic and photophysical properties of both ditopic ligand and metal ion enabled photomechanical response of the supramolecular organogels upon UV light irradiation through molecular design.

The development of supramolecular functional materials represents a challenging research topic of great current interest from both fundamental and application points of view. ${ }^{[1-2]}$ Precise management of weak intermolecular interactions (i.e., supramolecular approaches) in combination with the use of functional building blocks allows not only preparation of self-assembled and hierarchically organized complex systems, but also enables engineering of so-called "smart" materials, such as stimuli-responsive and adaptive soft materials. ${ }^{[3-7]}$ In this respect, we have recently reported on a novel class of supramolecular metallo-polymers based on bisterpyridine ditopic ligands functionalized with photoswitchable diazo-aryl groups. These polymers are capable of inducing solvent gelation affording to photo-responsive and

\footnotetext{
a. Institut de Science et d'Ingénierie Supramoléculaires (I.S.I.S.)

Université de Strasbourg, CNRS UMR 7006

8 allée Gaspard Monge, F-67083 Strasbourg, France

E-mail: mauro@unistra.fr

b. Département des Matériaux Organiques

Institut de Physique et Chimie des Matériaux de Strasbourg,

Université de Strasbourg, CNRS UMR 7504

23 rue du Loess, BP 43, F-67034 Strasbourg Cedex 2, France

E-mail: bellemin@unistra.fr

c. Université de Haute-Alsace, ENSCMu-Institut Donnet

Laboratoire de Photochimie et d'Ing_nierie Macromoléculaires

3 rue Alfred Werner, 68093 Mulhouse Cedex (France)

d. University of Strasbourg Institute for Advanced Study (USIAS)

5 allée du Général Rouvillois, F-67083 Strasbourg, France

† Electronic Supplementary Information (ESI) available: experimental details for the synthesis and characterization.
}

adaptive organo-[8] and hydrogels ${ }^{[9]}$. The use of zinc(II) salts with terpyridine (tpy) ligands, i.e. a coordination node with relatively high binding constant and kinetic lability, enabled high degree of polymerisation and dynamic metal-ligand bonds thus contributing to self-healing capability of the resulting gels.

Judicious choice of the nature and oxidation state of the coordinating metal centre embedded into the supramolecular architecture is expected to profoundly influence the properties of the resulting metallo-polymers and responsive materials. Indeed, on one hand, one might modify both kinetic lability and thermodynamic stability of the metal(tpy) ${ }_{2}$ unit (tpy $=$ $2,2^{\prime}: 6^{\prime}, 2^{\prime \prime}$-terpyridine type of ligands), which are known to control reversibility of the supramolecular self-assembly process between the tpy moiety and the metal cation (i.e., possibility to self-correct and self-repair) as well as solution equilibria, speciation (i.e., mono-tpy vs. bis-tpy species) and degree of supramolecular polymerisation, respectively. ${ }^{[10-17]}$ On the other hand, one may introduce added-value features including photophysical, magnetic and reversible redox properties upon employment of different and properly selected coordination metals. ${ }^{[18-20]}$

In particular, 3d transition metals $\mathrm{Fe}$ (II) and $\mathrm{Co}$ (II) are already known to form stable homoleptic complexes with terpyridine-type of ligands, being $\log \beta=20.9$ and 18.3 for $\mathrm{Fe}\left(\mathrm{tpy}^{2+}\right.$ and $\mathrm{Co}\left(\mathrm{tpy}^{2+}\right)^{2+}$, respectively. ${ }^{[21-22]}$ This ensures high degree of polymerisation in the metal-ligand supramolecular self-assembly process under practically-useful concentration conditions. These two transition metals are characterized by a lower kinetic lability than $\mathrm{Zn}$ (II) counterparts in the M-tpy interaction, the half-life of $\mathrm{M}(\mathrm{tpy})^{2+}$ species being in the order $\mathrm{Fe}(\text { tpy })^{2+}>\mathrm{Co}(\text { tpy })^{2+}>\mathrm{Zn}(\text { tpy })^{2+}\left(8400,50\right.$ and $0.1 \mathrm{~min}$ in $\mathrm{H}_{2} \mathrm{O}$, respectively). ${ }^{[23-24]}$

Aiming at the preparation of supramolecular materials in which photo-mechanical response could be finely controlled by molecular design of constituent building blocks, we investigated the effect of the variation of the metal(II) ion onto the photochemical behaviour of metallopolymeric gels based on photo-active ligand A and B (Scheme 1). Thus, we have chosen $\mathrm{Fe}$ (II) and $\mathrm{Co}$ (II) on the basis of thermodynamic and 
kinetic considerations abovementioned as well as on their different ground and excited state electronic properties that are expected to deeply influence the photo-switching ability of the azobenzene moiety encoded into the supramolecular architecture of the metallopolymers. ${ }^{[25]}$

Additionally, the choice of using $\mathrm{Fe}$ (II) and Co(II) allows us direct comparison with the previously reported systems based on zinc, given their relatively closer binding constants when compared to $4 \mathrm{~d}$ metals such as $\mathrm{Rh}$ (III) and Ru(II). Furthermore, we decided to use ligand $\mathbf{A}$ and $\mathbf{B}$ owing to high degree of conjugation between the photoactive moieties (i.e., azoaryl and phenylene-ethynylene, respectively) and the metal-ligand centre to keep highly coupled electronic interactions between them. ${ }^{[26]}$
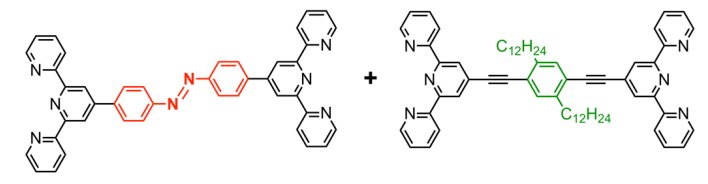

A

B

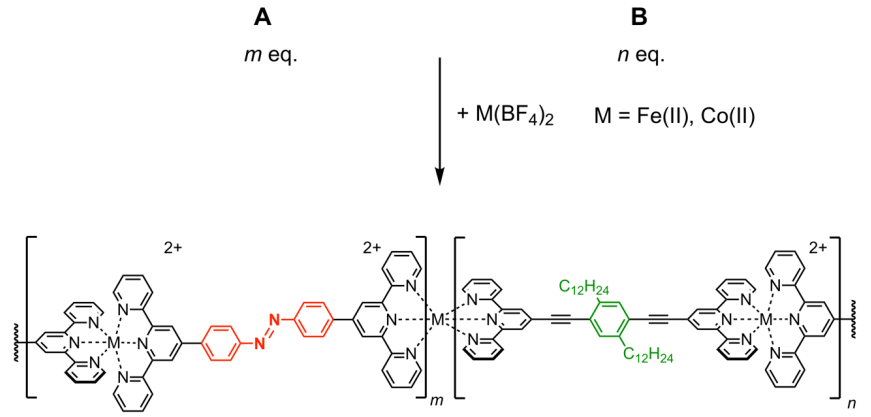

Scheme 1. Schematic synthetic pathway employed for the preparation of the $\mathrm{Fe}(\mathrm{II})$ and $\mathrm{Co}$ (II) telechelic metallopolymers with ditopic ligand $\mathbf{A}$ and $\mathbf{B}$. In the case of polymer Fe-P and Co-P the stoichiometry is $m=2$ and $n=1$.

The chemical structure of ligand $\mathbf{A}$ and $\mathbf{B}$ and the schematic synthetic pathway for the preparation of the supramolecular metallo-heteropolymers are displayed in Scheme 1.

Firstly, supramolecular polymerisation process by employing either $\mathrm{Fe}\left(\mathrm{BF}_{4}\right)_{2}$ or $\mathrm{Co}\left(\mathrm{BF}_{4}\right)_{2}$ as metal sources and a combination of ligand $\mathbf{A}$ and $\mathbf{B}$ has been followed in-situ by UVvisible spectroscopy to elucidate the possibility to form metallopolymer and to ascertain the position of the corresponding chemical equilibrium. Such a study was made possible by the peculiar features associated with the formation of each of the $\mathrm{M}(\mathrm{tpy})^{2+}$ species, where $\mathrm{M}$ is either $\mathrm{Fe}(\mathrm{II})$ or $\mathrm{Co}(\mathrm{II})$. It is important to notice that the $\mathrm{BF}_{4}$ - counter-anion (i.e., a non-coordinating one) has been chosen to favour formation of bis-terpyridine $\mathrm{M}(\mathrm{tpy})^{2+}$ nodes vs. monomeric $\mathrm{M}\left(\right.$ tpy) $\mathrm{X}_{2}$ (where $\mathrm{X}=$ anion or coordinating solvent molecule). The latter acts as chain termination species, thus lowering the degree of polymerisation. ${ }^{[27]}$

UV-visible spectra recorded upon addition of different aliquots of $\mathrm{Fe}^{2+}$ into a $\mathrm{CHCl}_{3}: \mathrm{CH}_{3} \mathrm{CN}$ 9:1 solvent mixture containing equimolar amount (concentration $=1 \times 10^{-5} \mathrm{M}$ ) of ditopic ligand $\mathbf{A}$ and $\mathbf{B}$ are displayed in Figure 1.
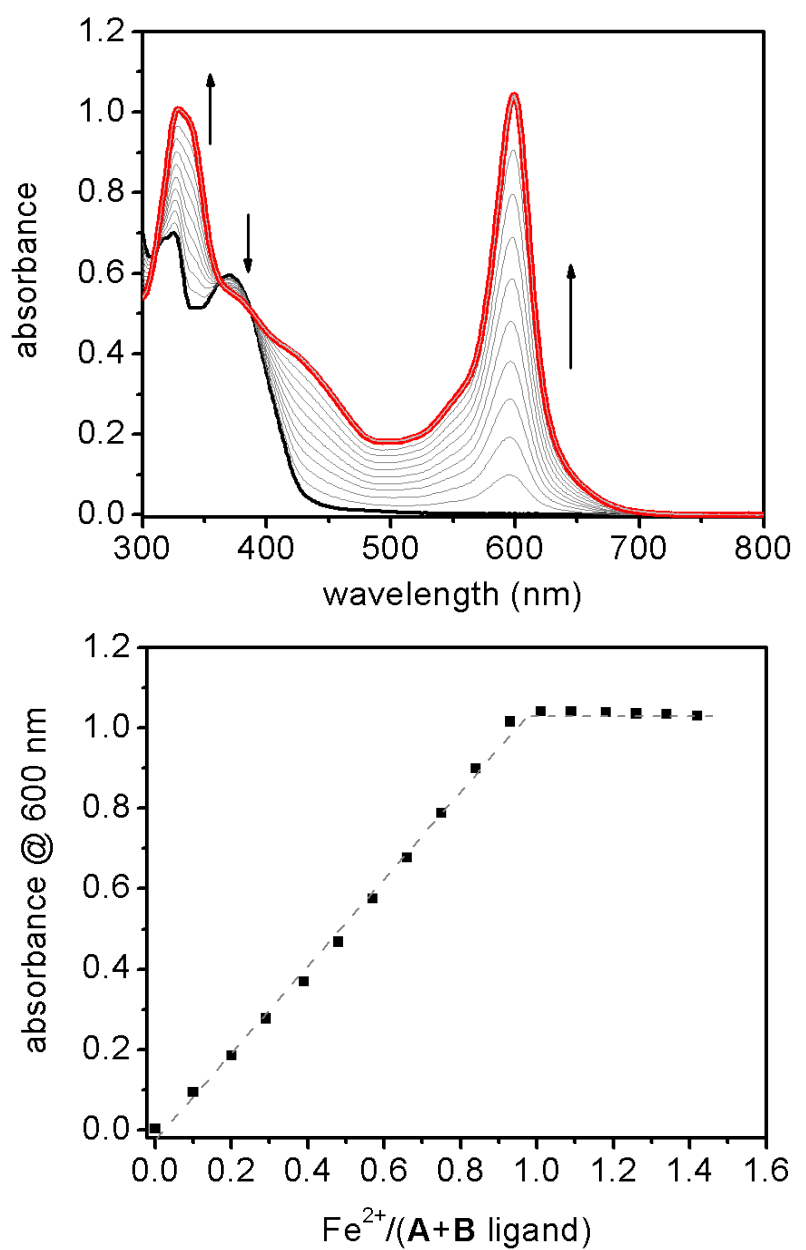

Figure 1. Titration of ligand $\mathbf{A}$ and $\mathbf{B}$ at concentration of $1 \times 10^{-5}$ $\mathrm{M}$ in $\mathrm{CHCl}_{3}: \mathrm{CH}_{3} \mathrm{CN}$ 9:1 with $\mathrm{Fe}\left(\mathrm{BF}_{4}\right)_{2}$ followed by UV-vis spectroscopy (top). The spectra corresponding to 0 and 1 eq. of $\mathrm{Fe}^{2+}$ added are displayed as black and red trace, respectively. Black arrows indicate the variation of the spectra features upon addition of the salt; plot of the absorbance variation recorded at $600 \mathrm{~nm}$ as a function of $\mathrm{Fe}\left(\mathrm{BF}_{4}\right)_{2}$ added (bottom). Dotted grey lines are only intended to guide the eye.

The absorption spectrum before addition of Fe(II) salt (black trace) is characterized by two intense bands centred at 326 and $370 \mathrm{~nm}$, corresponding to the overlapping singletmanifold ligand-centred $\left({ }^{1} \mathrm{LC}\right)$ transitions of both ligand $\mathbf{A}$ and B, i.e. ${ }^{1} \pi-\pi^{*}$ transitions of the terpyridine, phenyleneethynylene and azo moieties. The formally symmetryforbidden weak $n-\pi^{*}$ transition of the azo moiety is located at ca. $475 \mathrm{~nm}$. Upon addition of $\mathrm{Fe}^{2+}$ aliquots, the rise of a band at $\lambda_{\text {abs }}=330 \mathrm{~nm}$ is noticed, attributable to the ${ }^{1} \mathrm{LC}$ band of the $\pi$-conjugated ligand coordinated onto $\mathrm{Fe}^{2+}$ centres. Even more importantly, an intense band in the visible spectrum at $598 \mathrm{~nm}$ is observed, which is attributed to the ${ }^{1} \mathrm{MLCT}$ of the Fe(tpy) $)^{2+}$ type of complex. ${ }^{[28]}$ Such a band is bathochromically shifted when compared to the $\mathrm{Fe}(\mathrm{tpy})^{2+}$ benchmark complex as 
consequence of the more extended $\pi$-conjugated system in the tridentate ligand $\mathbf{A}$ and $\mathbf{B}$. Interestingly, the spectra display three isosbestic points at $\lambda_{\text {abs }}=313,362$ and $387 \mathrm{~nm}$ suggesting that coordinating moieties are arranged in only one fashion.

Plotting intensity of such ${ }^{1} \mathrm{MLCT}$ transition over increasing $\mathrm{Fe}^{2+}$ :(ligand $\left.\mathbf{A}+\mathbf{B}\right)$ ratio allowed following the metal-directed supramolecular polymerisation process. The plot clearly show a monotonic increase of the intensity associated to the ${ }^{1} \mathrm{MLCT}$ band upon increasing $\mathrm{Fe}^{2+}$ concentration until 1:1 ratio is reached indicating that in such condition complete metal coordination is achieved and the chemical equilibrium is shifted toward the formation of bis-terpyridine species (see Figure 1). As expected for a mechanism involving step-wise coordination of two terpyridine onto a $\mathrm{M}^{2+}$ ion in which $K_{2} \gg$ $K_{1}$, ${ }^{[29-30]}$ excess of metal cation did not result in an absorbance variation owing to the higher binding constant of $\mathrm{Fe}^{2+}$ for the formation of $\left[\mathrm{Fe}(\mathrm{tpy})_{2}\right]^{2+}$-type species. This demonstrate that the resulted metallopolymer is stable and do not undergo depolymerisation in the presence of excess of metal salts Moreover, the rigid nature of the telechelic ligands $\mathbf{A}$ and $\mathbf{B}$ points towards formation of linear supramolecular coordination polymers rather than self-assembled metallomacrocycles. ${ }^{[14,31]}$

Titration experiments carried out under identical condition in terms of solvent and ligands concentration, but employing $\mathrm{Co}\left(\mathrm{BF}_{4}\right)_{2}$ as metal source, afforded similar results, as shown in Figure S1 of the Electronic Supplementary Information (ESI). Upon addition of $\mathrm{Co}^{2+}$ aliquots, the resulting absorption spectra display appearance of a band at $331 \mathrm{~nm}$ attributable to the ${ }^{1} \mathrm{LC}$ transitions. Noteworthy, three much weaker bands appear at $\lambda_{\text {abs }} c a .400,460$ and $525 \mathrm{~nm}$ that can be ascribed to the MLCT and MC transitions of the Co(II)-ligand complex. ${ }^{[32]}$ Similarly to what observed for the formation of metallosupramolecular coordination polymer with $\mathrm{Fe}^{2+}$, and as one can expect on the basis of thermodynamic metal-ligand coordination constants for $\left[\mathrm{Co}(\mathrm{tpy})_{2}\right]^{2+}$, supramolecular polymerisation was completed at metal:ligand(A+B) ratio of 1 and excess of metal cation did not resulted into formation of dinuclear [Co(ditopic ligand)Co] ${ }^{4+}$ species (see Figure S1 of the ESI).

Metallopolymers were synthetized in bulk by refluxing a $\mathrm{CHCl}_{3}$ solution of ligand $\mathbf{A}: \mathbf{B}$ in a 2:1 ratio and stoichiometric amount of $\mathrm{M}\left(\mathrm{BF}_{4}\right)_{2}\left(\mathrm{M}=\mathrm{Fe}^{2+}\right.$ and $\left.\mathrm{Co}^{2+}\right)$ overnight, and the corresponding supramolecular polymers, namely Fe-P and Co$\mathbf{P}$, were obtained as orange and deep-violet solid, respectively (see Scheme 1, with $m=2$ and $n=1$ ). At a first attempt, fixed ratio of 2:1 between ditopic ligands $\mathbf{A}$ and $\mathbf{B}$ was chosen on the basis of our previous experiments. ${ }^{[8-9]}$ The details of the synthetic procedures are reported as ESI and the corresponding ${ }^{1} \mathrm{H}$ NMR spectrum for Fe-P displayed in Figure S2 of the ESI. We assume that the formation of metallopolymers results into random organization of both ligand $\mathbf{A}$ and $\mathbf{B}$ along supramolecular polymers chain. Both Fe$\mathbf{P}$ and Co-P metallopolymers dissolved in DMF were found capable to induce gelation upon addition of either $\mathrm{EtOH}$ or $\mathrm{CH}_{2} \mathrm{Cl}_{2}$ playing the role as nonsolvent, at overall polymer concentration as low as $0.12 \mathrm{wt} . \%$. This enabled investigation of the effect of electronic properties of the coordination metal on the photo-switching ability of the organogel.

Upon mixing either Fe-P or Co-P metallopolymer at concentration of $20 \mathrm{mg} \mathrm{mL}^{-1}$ in DMF and addition of a 20 -fold volume of non-solvent, highly coloured and stable gels were obtained within three hours at room temperature, indicating the high tendency of such supramolecular metallopolyelectrolytes to act as gelating agent though establishment of hydrophobic $\pi-\pi$ interactions between $\pi$-conjugated systems.

Mechanical properties of both Co-P and Fe-P gels prepared at $0.5 \mathrm{wt} . \%$ in DMF:EtOH 1:5 v/v where investigated by means of rheological experiments. As shown in Figure S3, a nonlinear dependency of storage and loss moduli, namely $\mathrm{G}^{\prime}$ and $\mathrm{G}^{\prime \prime}$, over strain was observed for both gels at a constant applied frequency of $1 \mathrm{~Hz}$, with yielding point at about $10 \%$ of strain. After such point, further increase of strain gave a sol-gel transition resulting in $G^{\prime \prime}$ exceeding $G^{\prime}$ values. Although the presence of more stable and less labile Fe(tpy $)^{2+}$ and Co(tpy $)^{2+}$ units within the supramolecular polymer skeleton of Co-P and Fe-P when compared to $\mathrm{Zn}$ (II) counterparts, ${ }^{[8-9]}$ thixotropic properties of the prepared gels were investigated to elucidate the effect of the interchain $\pi-\pi$ interactions onto gel stability. The results are displayed in Figure S4 in which low and high strain values were cycled over time. As shown, while at low $(0.5 \%)$ strain, storage modulus values exceeded the loss modulus, application of much higher (200\%) strain resulted in rupture of the gel network, most likely owing to the breakage of interchain $\pi-\pi$ interactions, and subsequent formation of the sol state $\left(G^{\prime \prime}>G^{\prime}\right)$. Interestingly, fast recovery of the gel state could be reversibly obtained when strain was set back to $0.5 \%$, confirming the thixotropic properties of Co-P and Fe-P gels.

In addition, the presence of photo-switching unit $\mathbf{A}$ within the metallopolymer structure prompted us to investigate the photochemical behaviour of the so-prepared organogels. Noteworthy, upon irradiation of gel samples with a LED lamp as UV light source $\left(\lambda_{\text {exc }}=365 \mathrm{~nm}\right)$, it was found out that Co-P gel showed neat light-induced contraction over an irradiation time of 12 hours, which is accompanied by syneresis (Figure 2). Indeed, electronic properties of the $\left[\mathrm{Co}(\mathrm{tpy})_{2}\right]^{2+}$ node are such, i.e. weak MLCT and MC band into the visible region, that they can not detrimentally affect the photo-switching of ligand $\mathbf{A}$ units.

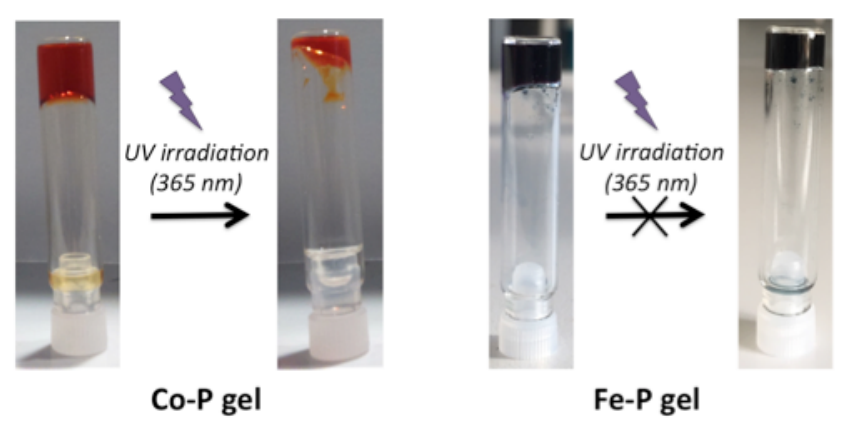


Figure 2. Photoresponse under UV irradiation with a continuous-wave LED lamp ( $\lambda_{\text {exc }}=365 \mathrm{~nm}$, irradiance: $21 \mathrm{~mW}$ $\mathrm{cm}^{-1}$ ) of the supramolecular gels containing either Co-P (left) or Fe-P (right). Organogels were prepared in DMF:EtOH (1:20 v/v) at concentration of 0.12 wt.\% of metallopolymer as the gelating agent.

On the other hand, no photomechanical response for gel made of Fe-P metallopolymer under identical excitation condition was observed even under an irradiation time as long as 24 hours (see Fig. 2). Such absence of gel response upon light-excitation into the azobenzene $\pi-\pi^{*}$ band is ascribed to ultrafast energy transfer (EnT) process from the excited donor azobenzene onto the acceptor MLCT band of the $\left[\mathrm{Fe}(\mathrm{tpy})_{2}\right]^{2+}$ moiety. ${ }^{[33-34]}$ This competitive photophysical process is thus expected to efficiently quench the photo-isomerization of the A unit by funnelling excitation energy into the iron(II)-tpy MLCT state, which in turn deactivates non-radiatively, most likely via $3,5 \mathrm{MC}$ state to the electronic ground state. At the present stage, it is important to notice that the photoswitching ability of the Co(II) metallopolymers enabled surface 2D nano-patterning and monitoring of the photochemical process at the solid-liquid interface by means of scanning probe techniques. ${ }^{[35]}$

In conclusion, two novel metallosupramolecular polymers have been synthetized in a straightforward synthetic pathway by using $\mathrm{Fe}$ (II) or $\mathrm{Co}$ (II) as coordination metal nodes for the telechelic ditopic ligands. The functional supramolecular metallo-polyelectrolytes, which include photo-isomerizable azoaryl units, are able to induce gelation of organic solvents resulting into thixotropic organogels. Proper tailoring the nature of the coordinating metal enabled the control of the photo-switching ability of the electronically coupled azo moiety. Thus, modulation of photomechanical response in supramolecular gel was achieved in an unprecedented manner. To the best of our knowledge, these represent the first examples of photoswitchable metallopolymeric organogels in which photomechanical response can be controlled by choice of the coordination metal. These results pave the way for better engineering of photo-responsive and adaptive supramolecular materials by chemical design.

The University of Strasbourg Institute for Advanced Study (USIAS) is kindly acknowledged for financially supporting this work.

\section{Notes and references}

1 J.-M. Lehn, Angew. Chem. Int. Ed., 2015, 54, 3276.

2 T. Aida, E. W. Meijer and S. I. Stupp, Science, 2012, 335, 813;

3 S. S. Babu, V. K. Praveen and A. Ajayaghosh, Chem. Rev., 2014, 114, 1973;

4 C. D. Jones and J. W. Steed, Chem. Soc. Rev., 2016, 45, 6546;

5 M. J. Mayoral Muñoz and G. Fernandez, Chem. Sci., 2012, 3, 1395;

6 M. A. Cohen Stuart, W. T. S. Huck, J. Genzer, M. Müller, C. Ober, M. Stamm, G. B. Sukhorukov, I. Szleifer, V. V. Tsukruk, M. Urban, F. Winnik, S. Zauscher, I. Luzinov and S. Minko, Nature Mater., 2010, 9, 101;
7 M. Häring and D. Díaz-Díaz, Chem. Commun., 2016, 52, 13068;

8 E. Borré, J.-F. Stumbé, S. Bellemin-Laponnaz and M. Mauro, Angew. Chem. Int. Ed., 2016, 55, 1313;

9 E. Borré, S. Bellemin-Laponnaz and M. Mauro, Chem. - Eur. J., 2016, 52, 18718;

10 A. Winter and U. S. Schubert, Chem. Soc. Rev., 2016, 45, $5311 ;$

11 E. C. Constable, K. Harris, C. E. Housecroft and M. Neuburger, Dalton Trans., 2011, 40, 1524;

12 M. Chiper, M. A. R. Meier and J. M. Kranenburg, Macromol. Chem. Phys., 2007, 208, 679;

13 R. Shunmugam, G. J. Gabriel, K. A. Aamer and G. N. Tew, Macromol. Rapid Commun., 2010, 31, 784;

14 R. Dobrawa and F. Würthner, J. Pol. Sci : Part A : Pol. Chem., 2005, 43, 4981;

15 F. Würthner, C.-C. You and C. R. Saha-Müller, Chem. Soc. Rev., 2004, 33, 133;

16 G. R. Whittel, M. D. Hager and U. S. Schubert, I. Manners, Nature Mater., 2011, 10, 176;

17 R. J. Wojtecki, M. A. Meador and S. J. Rowan, Nature Mater., 2011, 10, 14;

18 R. Sakamoto, K.-H. Wu, R. Matsuoka, H. Maedaa and H. Nishihara, Chem. Soc. Rev., 2015, 44, 7698;

19 A. El-Ghayoury, A. P. H. J. Schenning and E. W. Meijer, J. Pol. Sci. Part A: Polym. Chem., 2002, 40, 4010;

20 S.-C. Yu, C.-C. Kwok, W.-K. Chan and C.-M. Che, Adv. Mater., 2003, 15, 1643;

$21 \beta$ is defined as the overall binding constant for the formation of the bis-trischelate metal complex;

22 R. H. Holyer, C. D. Hubbard, S. F. A. Kettle and R. G. Wilkins, Inorg. Chem., 1966, 5, 622;

23 S. M. Munzert, G. Schwarz and D. G. Kurth, Inorg. Chem., 2016, 55, 2565;

24 R. Hogg and R. G. Wilkins, J. Chem. Soc., 1962, 341;

25 T. Yutaka, I. Mori, M. Kurihara, N. Tamai, H. Nishihara, Inorg. Chem., 2003, 42, 6306;

26 M. Burnworth, J. D. Mendez, M. Schroeter, S. J. Rowan and C. Weder, Macromolecules, 2008, 41, 2157;

27 Detailed speciation studies in solution were previously reported and they can be found in Dalton Trans. 2011, 40, 11441 ;

28 Y. Bodenthin, G. Schwarz, Z. Tomkowicz, T. Geue, W. Haase, U. Pietsch and D. G. Kurth, J. Am. Chem. Soc., 2009, 131, 2934;

$29 K_{1}$ and $K_{2}$ are defined as the binding constant corresponding to tpy $+\mathrm{M}^{2+} \rightleftarrows[\mathrm{M}(\text { tpy })]^{2+}$ and tpy $\left.+[\mathrm{M}(\text { tpy })]^{2+} \rightleftarrows \mathrm{M}(\text { tpy })_{2}\right]^{2+}$ coordination event, respectively;

30 V. A. Friese, D. G. Kurth, Coord. Chem. Rev., 2008, 252, 199;

31 E. C. Constable, C. E. Housecroft, M. Neuburger, S. Schaffner, C. B. Smith, Dalton Trans., 2005, 2259;

32 J. S. Judge, W. A. Baker Inorg. Chim. Acta, 1967, 1, 68;

33 T. Yutaka, I. Mori, M. Kurihara, N. Tamai and H. Nishihara, Inorg. Chem., 2003, 42, 6306;

34 G. Auböck and M. Chergui, Nat. Chem., 2015, 7, 629;

35 manuscript submitted. 